\title{
Robust Method for Detecting the HRI Device using RANSAC
}

\author{
M.S. Gil ${ }^{a}$, M.S. Kang ${ }^{a}$, Y.S. Lee ${ }^{b}$, S.H. Lee ${ }^{b}$, S.H. Kim ${ }^{b}$ and C.S. Han ${ }^{\mathrm{c}}$ \\ ${ }^{a}$ Dept. of Mechatronic, Eng., Hanyang University, Republic of Korea \\ ${ }^{b}$ Dept. of Mechanical, Eng., Hanyang University, Republic of Korea \\ ${ }^{c}$ Dept. of Robot, Eng., Hanyang University, Republic of Korea \\ E-mail: msgill798@gmail.com, wowmecha@gmail.com, pacheon76@hanyang.ac.kr, \\ hopezic@gmail.com, shkim83@ hanyang.ac.kr, cshan@ hanyang.ac.kr
}

\begin{abstract}
-
Installation work of large inner/outer wall panel glasses increases the labor load and stress of workers and causes the danger of such accidents as falling and crane overturning. To solve this problem, Gil designed the easy handling robot system which is composed of mobile system, 5-DOF manipulator system and HRI (Human-Robot Interface) device included HRC (Human-Robot Cooperation) algorithm. During glass installation work using this robot system, detecting the position of the HRI device has great influence on the work efficiency. While working there, the construction worker placed the HRI device on panel glass randomly. Also there are many factors that disturb the detecting the HRI device. In this paper describes a method for detecting the HRI device robustly. To robustly find the HRI device, in this paper, applied the RANSAC (RANdom SAmple Consensus) and LSF (Least Square Fit) algorithm. And sensor module for detect the HRI device is composed of IR (Infrared Ray) sensor and RC servo motor. The distance between the sensor module and the circle-shaped HRI device is utilized for detecting this device. In this paper, to verify the robust method, performed laboratory experiment: for detecting the HRI device randomly placed on, for finding the HRI device put with a square-shaped disturbance.
\end{abstract}

Keywords -

RANSAC; LSF; Human-Robot Interface device

\section{Introduction}

Installation of large inner/outer wall panel glasses such as curtain wall installation work in construction sites is carried out at the border between the inside and outside of a building. This kind of work increases the labor load and stress of workers and the danger of such accidents as falling and crane overturning. Until now, such large glass finishing materials have been installed by workers. Except for the movement of glasses, all the work processes including assembly and installation have depended on workers [1]. This is because there is a limitation in completely automating glass installation works (access to the installation position and fitting) in the changeable environment of construction sites. Furthermore, as the high-rise buildings become larger, it is an inevitable trend that such construction materials become larger and heavier, and installations of these materials have naturally increased. Therefore, as a measure to guarantee the safety of construction workers and shorten construction period, semi-automation systems based on human-robot cooperation (HRC) techniques instead of full automation are being developed and applied [2,3].

OKTOPUS by Materials Handling Corp. in Australia, Mobile Ergonomic Handler by Arlington Equipment Corp. in the U.S., Geko \& Glass Robot Hire by GGR Corp. in the U.K., and KS Robot 280 by K.Schulten $\mathrm{GmbH} \&$ Co.KG. In Germany are representative glazing robot systems based on humanrobot cooperation technology [4-7]. Similarly, Yu et al. in Korea developed a curtain wall robot with a 3-DOF manipulator attached to the end-effector of a miniexcavator [8]. For these robots, the operator manipulates the joints of the robots. If unskilled operator uses this robot manipulation method, however, it takes long time to work and has a high likelihood of accidents. To address this problem, Lee et al. developed a ceiling glass installation robot with 6-DOF F/T sensors so that operator can directly operate the robot from the robot end-effector [9]. Furthermore, Lee applied force control to promote the safety of workers and protect glasses when operator works from the robot end-effector [10]. Force control transmits the repulsive power of materials against the environment to the operator so that the operator can safely operate the robot for such tasks as assembly. However, additional problems occurred in actual application such as sensor cost, calibration of the weight of heavy objects, and the separation of force and torque. Thus, Gil et al. proposed a robot control method in which the operator defines the rotational axis of the 
panel glass and the motion of glasses is determined by the resultant force applied to this axis and a point of action on the panel glass [11]. And Gil et al. designed an easy handling robot (EHR) system for installing the panel glass such as a curtain-wall. EHR is composed of mobile system, 5-DOF manipulator system and HRC (human-robot cooperation) algorithm. This robot system is available to move and rotate in a confined space. Also an operator, using a HRI (human-robot interface) device and robot manipulator, is available to work easily [12].

In this paper describes a robust method for detecting this HRI (human robot interface) device. Chapter 2 in this paper shows the summary of the HRC (humanrobot cooperation) algorithm and the HRI (human-robot interface) device. Chapter 3 describes detecting sensor module and robust detecting method. Chapter 4 shows the experimental result of this method.

\section{Human-robot interface device}

\subsection{Human-robot cooperation algorithm}

The HRC algorithm proposed by Gil et al. is described below. In general, the fundamental study of the HRC algorithm is the motion of a rigid body in Cartesian space. To make it identical to the actual glass installation method by construction workers, the instantaneous axis of rotation (IoR) and the point of action (PoA) of the glass are defined. During the installation work by construction worker, the panel glass has an IoR and PoA and the position of these axis change continuously. Gil et al. analysed the motion of panel glass during installation work by construction worker and typically deduced the 3 position of IoR. Figure 1 (a) shows the position of IoR which is located on center of panel glass. At this time, the position of PoA is determined by a resultant force of two workers. When firstly point contact is occurred between panel glass and installation plane, such as figure 1 (b), the position of IoR is located on this point. And PoA is randomly positioned on panel glass except this point. Figure 1 (c) shows the line contact by two point contact. When occurring line contact between panel glass and installation plane, the position of IoR is located on edge of panel glass. And PoA is randomly positioned on panel glass except this edge line.

When the IoR is located at infinity, the panel glass has a linear motion. But when occurring the contact between the panel glass and installation plane (point contact, line contact), it has a rotational motion. Because the operator, for maintaining the point and line contact, push or pull the panel glass.

HRC algorithm is a control method that panel glass has a linear and rotational motion by a predefined rotational axis and PoA. And a control input is two wor-

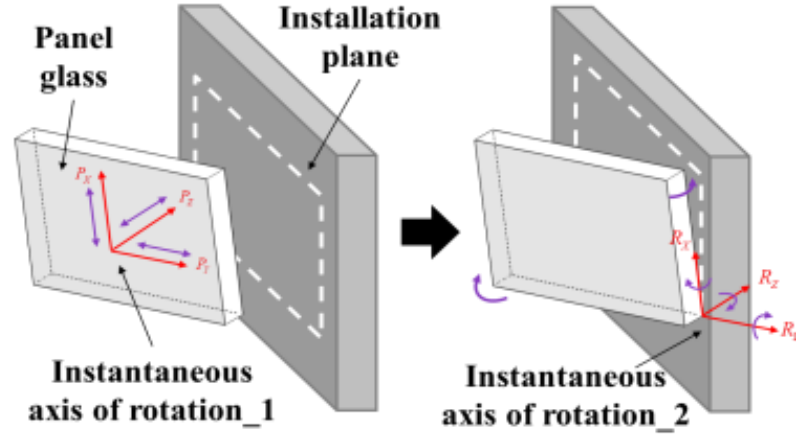

(a)

(b)
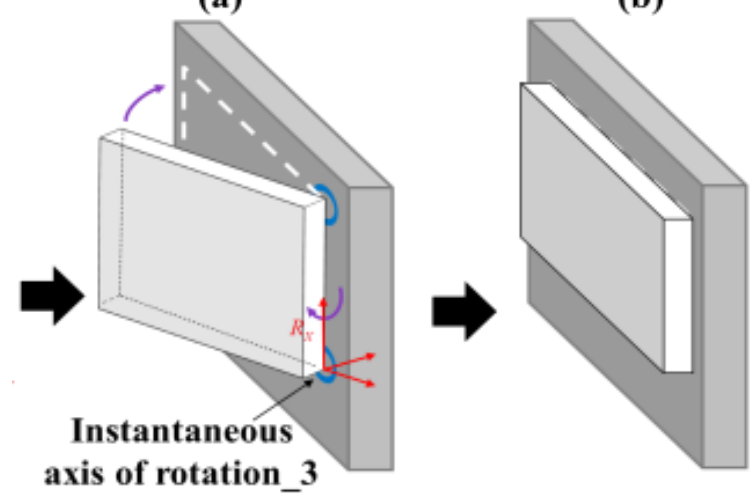

(c)

(d)

Figure 1. The position of IoR for glass installation work

-ker's force. . In HRC algorithm, it is assumed that the panel glass has a linear and rotational motion when construction worker apply force to rotational axis and PoA. Simultaneously, if two construction worker applies force to these axis, the panel glass has a composite motion.

The HRI (human-robot interface) device attached on 3 -axis load cell is an input device for robot motion. And the location of this device is same as a rotational axis and PoA of the panel glass. Two construction workers randomly put these devices on panel glass.

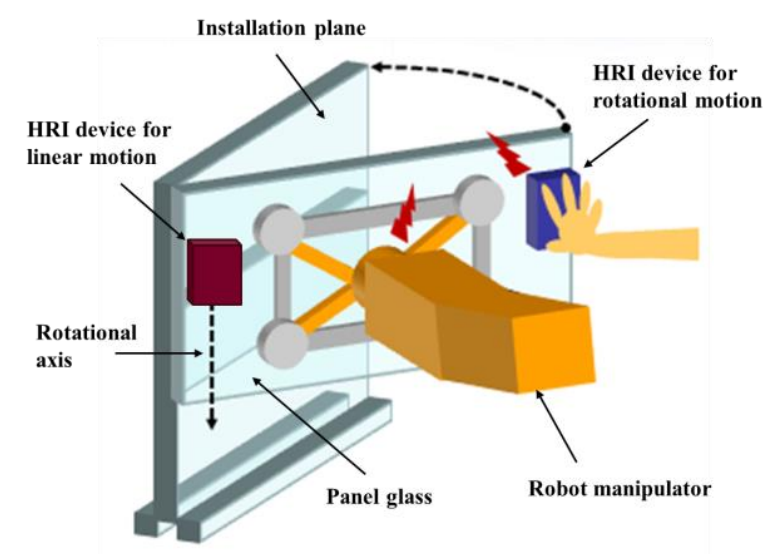

Figure 2. The HRI device for HRC algorithm 
Figure 2 shows the HRI devices for HRC algorithm. In this figure, two HRI devices put on panel glass. These devices define the position of rotational axis and PoA, also it measure the worker's force and transmit to the robot manipulator by electronic signal. This figure presents when panel glass has a rotational motion.

\subsection{Detailed design of human-robot interface device}

The HRI device, which measure the operator's force, is composed of a handle, a 3 -axis load cell and a suction device (figure 3). To grip the HRI device, a handle part is considered. And 3-axis load cell measure the operator's force. So, to easily put the HRI device on the panel glass, a suction device is made up of a lever and a sucking disk. The sucking method is a screw. That is, while the lever rotates, the sucking disk expands. As a result, the IMD is attached to the surface of the panel glass. To find the IMD easily, a detecting section is designed in the circle shape with a $100 \mathrm{~mm}$ diameter The height of the IMD is $150 \mathrm{~mm}$ and the allowed weight of the sucking disk is $80 \mathrm{~kg}$.

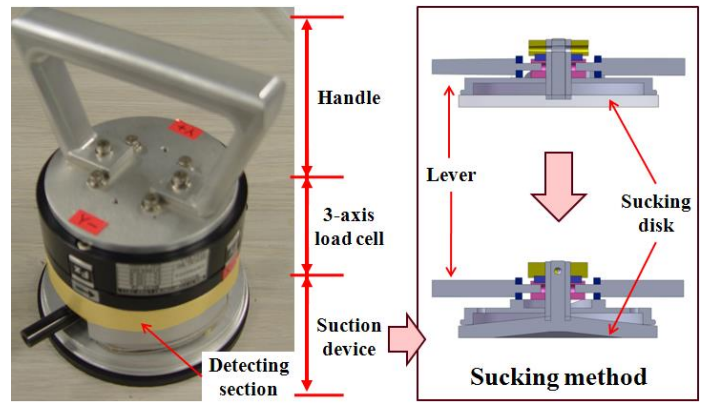

Figure 3. Detailed design of HRI device

\section{Robust detecting method}

\subsection{Detecting sensor module and method}

In this paper, to detect the HRI device which is randomly placed on the panel glass, detecting sensor module is applied. This sensor module is composed of

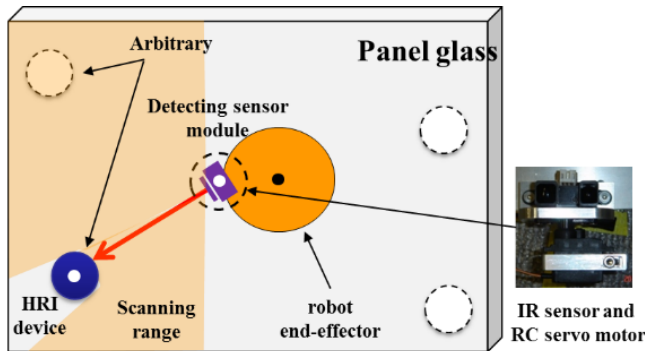

Figure 4. Detecting sensor and method
IR sensor and RC servo motor. IR sensor measures the distance between robot end-effector and contour of HRI device. Figure 4 presents the detecting sensor module and method.

\subsection{Robust detecting method}

In case of scanning the contour of HRI device using IR sensor, unexpected disturbance can be detected such as operator's hand and reflected light. Thus, it must be robustly found the contour by detecting sensor module. In this paper, using the RANSAC (RANdom SAmple Consensus) and LSF (Least Square Fit) algorithm, it can be found the IMD's contour by sensor module robustly. RANSAC algorithm is an iterative method to estimate parameters of a mathematical model from a set of observed data which contains outliers or disturbance [13]. In this paper, it estimate the mathematical model of HRI device from the observed data by sensor module. The robust detecting method is following.

1. Gathering the observed data in twice (clockwise and anticlockwise direction)

2. Apply the RANSAC algorithm to sampled data and select the data group $\left(\mathrm{G}_{\mathrm{d}}\right)$

3. Apply the LSF algorithm to these data group $\left(\mathrm{G}_{\mathrm{d}}\right)$ and estimate the circle equation.

4. Estimate the center position from this circle equation

5. Deduce the center position of the HRI device by mean value.

Firstly, the method for gathering the sample data is following. In figure $5, \mathrm{RC}$ servo motor is controlled that it turns the IR sensor from 0 (degree) to 160 (degree) in clockwise ( (1) direction and anticlockwise ( (2) direction by 1 degree. And it gathers the IR sensor data in 12 times each degree and calculate the average. At th-

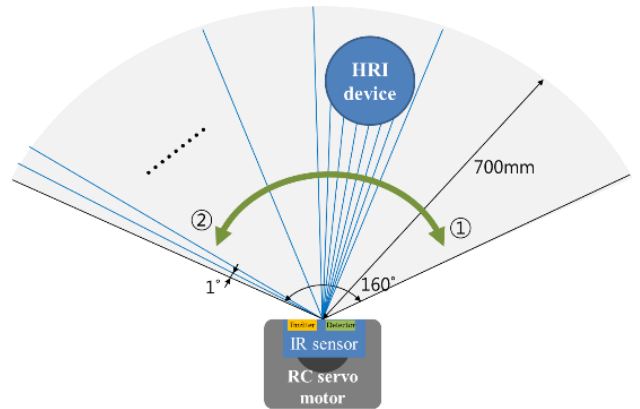

Figure 5. RC servo motor control method 
-is time, considering the timing delay between first output and first measurement, it gathers the data after the RC motor is rotated in 1 degree. Also it is defined that the HRI device is located from $200 \mathrm{~mm}$ to $700 \mathrm{~mm}$ because of IR sensor's linearity

In gathered data, this set contains both inliers (points which approximately can be fitted to a parameterized model) and outliers. To select the inliers from gathered data, RANSAC algorithm is applied. RANSAC achieves its goal by iteratively selecting a random subset of the original data. RANSAC is divided from hypothetical generation and evaluation. To achieve the goal, firstly, it selects the sample data randomly. And it estimate the model (circle model) using sampled data. Then it calculates the error of data with respect to estimation. After it count number of inlier candidate, if sufficiently many points have been classified as part of the set then keep this set. After enough number of iteration, it return the final estimation. Figure 6 shows RANSAC algorithm. For applying RANSAC, N (iteration number) and $\mathrm{T}$ (boundary line between inlier and outlier) parameter must be defined. In this paper, for detecting the HRI device, it is defined that $\mathrm{N}$ is 50 sample and $\mathrm{T}$ is $10 \mathrm{~mm}$.

After applying the RANSAC algorithm, applying the LSF algorithm, estimate the center position of IMD. The LSF algorithm is based on minimizing the mean square distance from the fitting curve to data points [14]. Given $\mathrm{n}$ points $\left(\mathrm{x}_{\mathrm{i}}, \mathrm{y}_{\mathrm{i}}\right), 1 \leq \mathrm{i} \leq \mathrm{n}$, the objective function is defined by

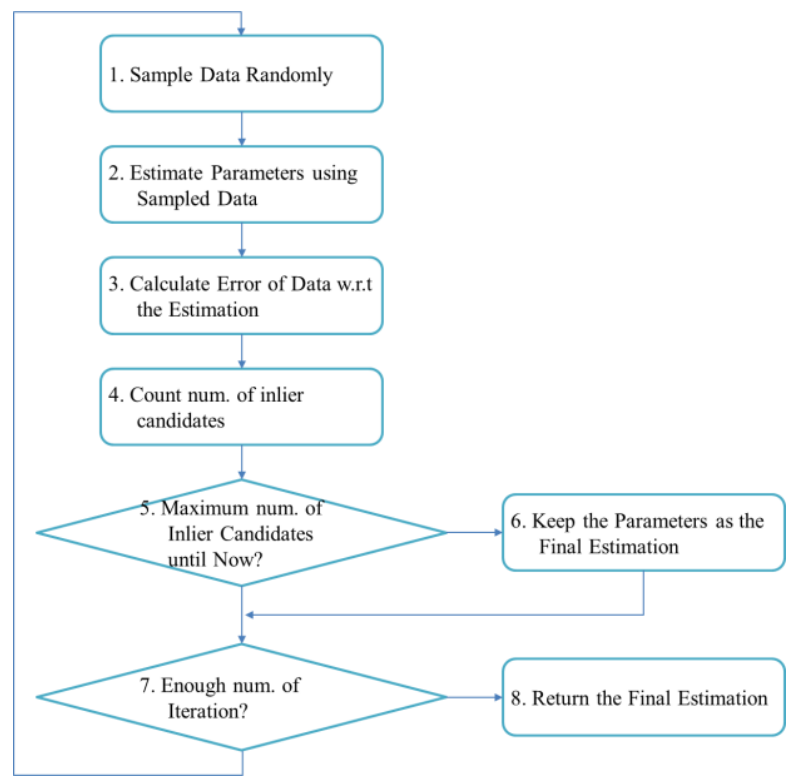

Figure 6. RANSAC algorithm

$$
\mathrm{F}=\sum_{i=1}^{n} d_{i}^{2}
$$

Where, $d_{i}$ is the Euclidean (geometric) distance from the points $\left(\mathrm{x}_{\mathrm{i}}, \mathrm{y}_{\mathrm{i}}\right)$ to the curve. When fitting circles, one parameterizes those by the equation

$$
(x-a)^{2}+(y-b)^{2}=R^{2}
$$

where $(\mathrm{a}, \mathrm{b})$ is the center and $\mathrm{R}$ is the radius.

Figure 7 presents the position of HRI device's contour and center. Where, the red line and points are the inlier data group during clockwise direction and the blue line and points are during anticlockwise direction. In figure 7, $\circ$ is center of circle (contain blue and red), $\mathrm{x}$ is inlier data group in clockwise direction and $\otimes$ is inlier data group in anticlockwise direction.

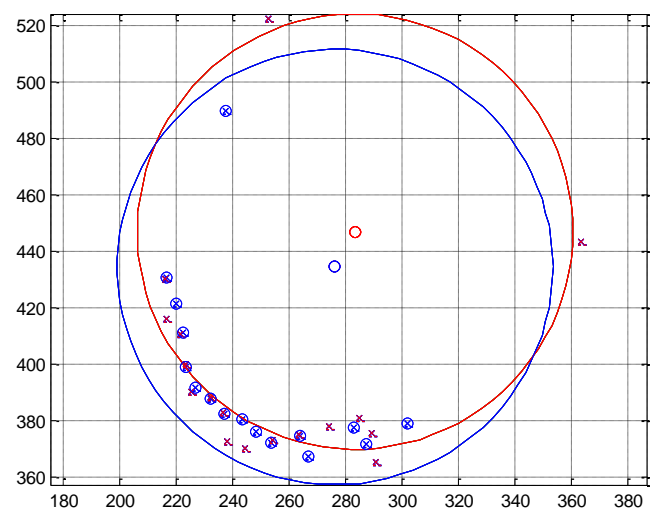

Figure 7. Applied the LSF algorithm

Finally, using the average of two center points in figure 7, it estimate the center of HRI device.

\section{Experiment}

To verify the robust detecting method, this chapter shows laboratory experiment. Two test is performed. First, the HRI device is placed on specific position. Aft-

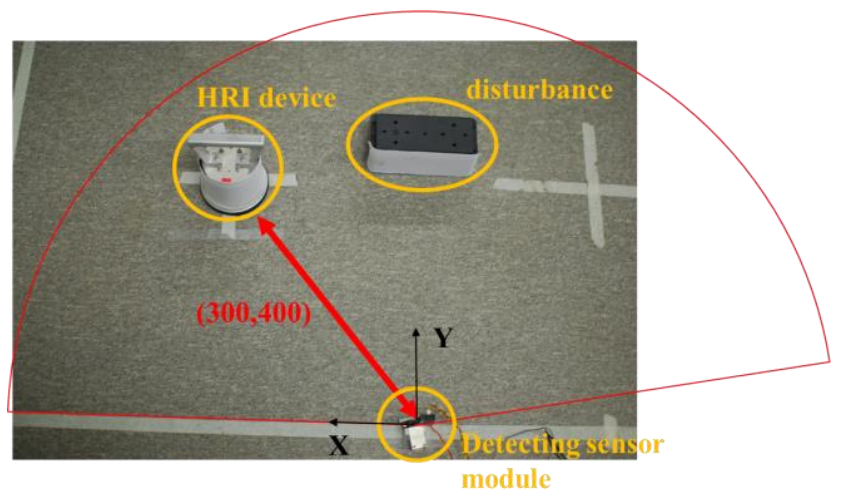

(a) The system configuration for experimental test 


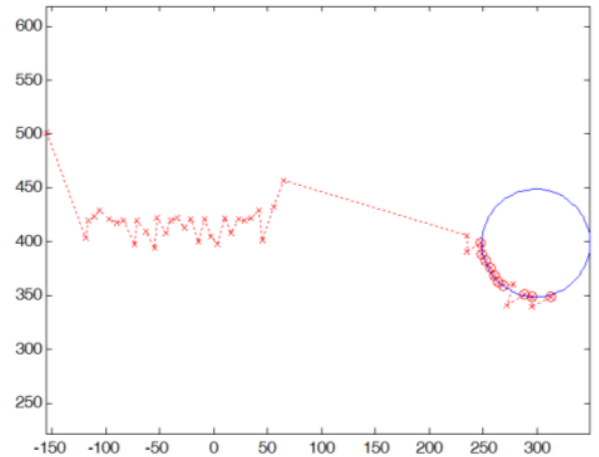

(b) The result of test

Figure 8 . The system configuration and result of test

-er many times scanning, it is to verify the performance of detecting algorithm. In second test, the HRI device is scanned with disturbance which is placed on different position.

Test -1 : Detecting sensor module is located on the origin point. And the HRI device is located in $\mathrm{x}=$ $300 \mathrm{~mm}, \mathrm{y}=400 \mathrm{~mm}$. the sensing range of detecting sensor module is between $200 \mathrm{~mm}$ and $700 \mathrm{~mm}$. (figure 8 (a))

Table 1 The result of experiment (mm)

\begin{tabular}{|ccccc|}
\hline & \multicolumn{2}{c}{$\mathrm{X}$} & \multicolumn{2}{c|}{$\mathrm{Y}$} \\
\cline { 2 - 5 } & $\begin{array}{c}\text { clock- } \\
\text {-wise }\end{array}$ & $\begin{array}{c}\text { anticlock- } \\
\text {-wise }\end{array}$ & $\begin{array}{l}\text { clock- } \\
\text {-wise }\end{array}$ & $\begin{array}{c}\text { anticlock- } \\
\text {-wise }\end{array}$ \\
\hline 1 & 283.39 & 276.37 & 417.04 & 437.47 \\
2 & 270.45 & 269.75 & 418.18 & 424.64 \\
3 & 292.35 & 269.79 & 419.85 & 426.68 \\
4 & 275.44 & 275.87 & 415.85 & 427.85 \\
5 & 296.52 & 269.25 & 419.75 & 428.74 \\
\hline average & 282.01 & 272.20 & 418.13 & 429.07 \\
\hline
\end{tabular}

In figure $8(\mathrm{~b}), \mathrm{x}$ red points are outliers and o points are inliers. The result of test the average error for $\mathrm{X}$ axis is about within $18 \mathrm{~mm}$ in clockwise direction and $28 \mathrm{~mm}$ in anticlockwise. And the average error for $\mathrm{Y}$ axis is about within $20 \mathrm{~mm}$ in clockwise direction and $30 \mathrm{~mm}$ in anticlockwise (table 1).

Test - 2: In figure 9 (a), this case shows that the circle shape and the square shape are touched with each other, then the sensor data is continuous. In figure 9 (b), this case present that the circle shape is hidden behind the square shape. In the last case (c) shows that the circle shape is away from the square shape.

In figure 10 , each case presents test result in clockwise direction and anticlockwise. In figure, the blue circle is contour of the HRI device. And red points are raw data from detecting sensor module.

In case of figure 9 (a) and (c), the HRI device is not covered by disturbance. Therefore, the data for device's contour can be gathered wholly. After applying the algorithm, it can be found the contour of the HRI device with small error such as figure 10 (a) and (c). But in case of figure 9 (b), the HRI device is hidden behind the dist-

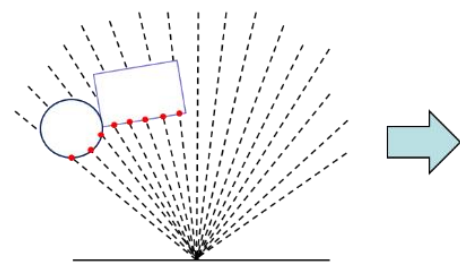

(a) Case - 1

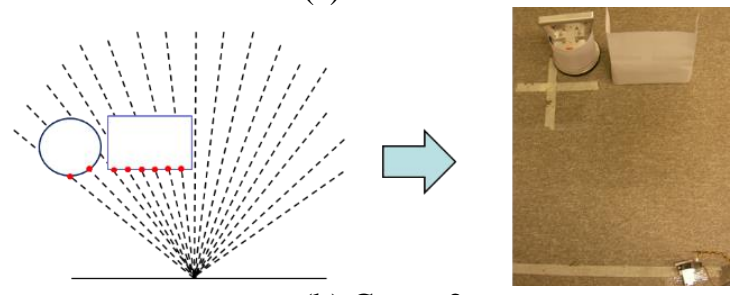

(b) Case - 2
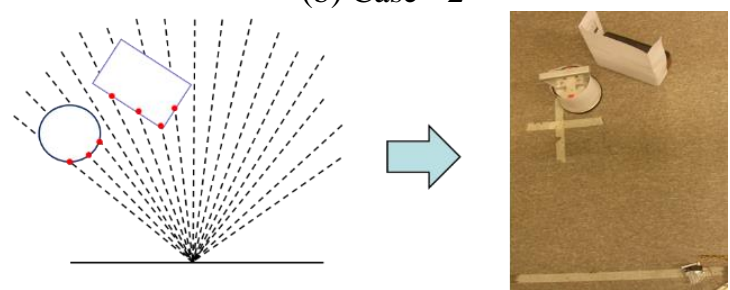

(c) Case - 3

Figure 9. Test configuration for detecting robustly
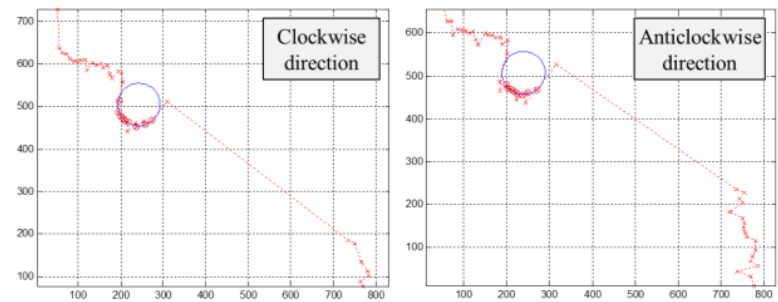

(a) Test result of case - 1
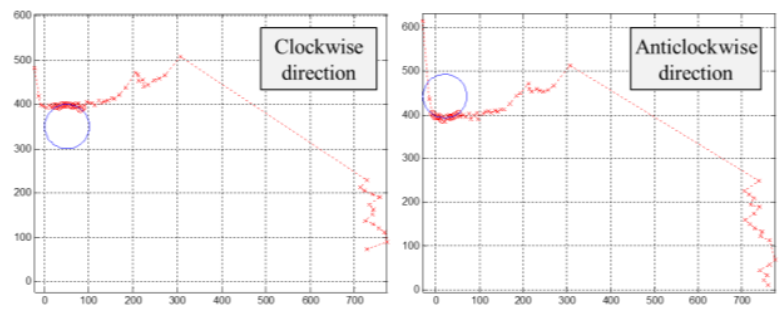

(b) Test result of case - 2

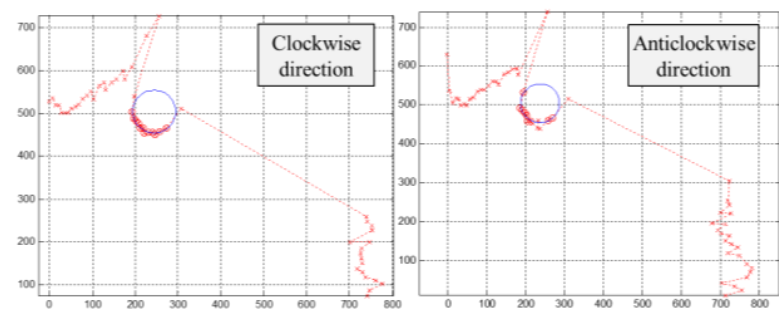


(c) Test result of case - 3

Figure 10. Test result for detecting robustly -urbance because the detecting sensor module is in Polar coordinate. After applying the algorithm, it cannot be found the contour of the HRI device such as figure 10 (b). The result of the test, the detecting algorithm applied RANSAC and LSF can robustly find the HRI device with small error but in some cases cannot find such as hidden. Also though it is applied to same sample data group, the result can be different. This is because the RANSAC selects the sample data randomly.

\section{Conclusion}

Installation work of large inner/outer wall panel glasses increases the labor load and stress of workers and the danger of such accidents as falling and crane overturning. To solve this problem, Gil designed the easy handling robot system which is composed of mobile system, 5-DOF manipulator system and HRI device included HRC algorithm.

In this paper describes a method for detecting the HRI device robustly. To robustly find the HRI device, in this paper, propose the algorithm containing RANSAC and LSF. And the detecting sensor module for the HRI device is composed of IR sensor and RC servo motor. The IR sensor measures the distance between robot end-effector and contour of the HRI device. The RC servo motor is controlled that it turns the IR sensor from 0 (degree) to 160 (degree) in clockwise direction and anticlockwise direction by 1 degree.

To verify the robust detecting method, this chapter performed laboratory experiment. Two test is performed First test is aimed to performance of algorithm and second test is aimed to robustness. Test result, the performance of algorithm is that it has a small error (clockwise: within $20 \mathrm{~mm}$, anticlockwise: within $30 \mathrm{~mm}$ ) and the robustness is that in case the HRI device is not hidden by disturbance, it can be found the contour. Also though it is applied to same sample data group, the result can be different. This is because the RANSAC selects the sample data randomly.

\section{Acknowledgement}

This research was funded by Building-façade Maintenance Robot research Center, supported by Korea Institute of construction and Transportation Technology Evaluation and Planning under the Ministry of Land, Transport, and Maritime Affairs (MLTM), the MKE (The Ministry of Knowledge Economy), Korea, Technology Innovation Program (10040180).

\section{References}

[1] Carlos Balaguer and Mohamed Abderrahim, "Trends in Robotics and Automation in Construction", 2008, ISBN 978-953-7619-13-8, InTech

[2] Fukuda T., Fujisawa Y., Muro E., Hoshino H., Miyazaki T., Uehara K., Ohtsubo K. and Mikami T., "A New Robotic Manipulator in Construction based on Man-Robot Cooperation Work", Proc. Of the 8th ISARC, pp. $2439 \sim 2445$.

[3] Fukuda T., Fujisawa Y., Kosuge K., Arai F., Muro E., Hoshino H., Miyazaki K., Ohtsubo K. and Uehara K., "Manipulator for Man-Robot Cooperation", International Conference on Industrial Electronics, Control and Instrumentation, Vol. 2, pp. 996 1001.

[4] OKTOPUS, www.materialshandling.com.au/, Australia, Materials Handling Co.

[5] Mobile Ergonomic Handler, www.gotoartech.com, U. S.A, Arlington Equipment Co.

[6] Geko \& Glass Robot Hire, www.ggrglass.co.uk, Engla nd, GGR Co.

[7] KS 280, http://www.ksschulten.com/de/index.html, Germany, K. Schulten Gmbh \& Co.KG

[8] Seung-Nam Yu, Seung-Yel Lee, Chang-Soo Han, Kye-Young Lee and Sang-Heon Lee, "Development of the curtain wall installation robot: Performance and efficiency tests at a construction site", Autonomous Robots, pp.281-291, 2007

[9] Seungyeol Lee, Myeongsu Gil, Kyeyoung Lee, Sangheon Lee and Changsoo Han, "Design of a Ceiling Glass Installation Robot", International Symposium on Automation and Robotics in Construction (ISARC 2007), pp.247-252, 2007

[10] Seung-Yeol Lee, Kye Young Lee, Sang Heon Lee, Jin Woo Kim, Chang Soo Han, "Human-robot cooperation control for installing heavy construction materials", Autonomous Robots, Vol. 22, pp. 305-319, 2007

[11] Myeong-Su Gil, Min-Sung Kang, Seunghoon Lee, Hee-Don Lee, Kyoo-sik Shin, Ji-Yeong Lee, Chang-Soo Han, "Installation of heavy duty glass using an intuitive manipulation device", Automation in Construction, Vol. 35, pp. 579-586, 2013

[12] M.S. Gil, S.H. Kim,H.G. Kim, M.S. Kang, C.S. Han "An easy handling system for installing heavy glass using human robot cooperation" Gerontechnology, Vol.11, No.2, 371-376, 2012

[13] M.A. Fischler and R.C. Bolles. "Random sample consensus : A paradigm for model fitting with applications to image analysis and automated 
The 31st International Symposium on Automation and Robotics in Construction and Mining (ISARC 2014)

cartography", Communications of the ACM, Vol.

24, No. 6, pp.381-395, 1981

[14] N. CHERNOV and C.LESORT, "Least Squares Fitting of Circles", Journal of Mathematical Imaging and Vision, Vol. 23, 239-252, 2005 